\title{
Driving Resumption After Critical Illness: A Survey and Thematic Analysis of Patient Experience and Process
}

Joel Meyer ( $\sim$ Joel.Meyer@gstt.nhs.uk)

St. Thomas' NHS Foundation Trust, London https://orcid.org/0000-0003-3303-3514

Natalie Pattison

University of Hertfordshire

Chloe Apps

Guy's and St Thomas' NHS Foundation Trust https://orcid.org/0000-0002-4855-4243

Melanie Gager

Royal Berkshire NHS Foundation Trust

\section{Carl Waldmann}

Royal Berkshire NHS Foundation Trust

\section{Research}

Keywords: Driving, Critical illness, Critical Care, Recovery, Intensive care

Posted Date: October 9th, 2020

DOI: https://doi.org/10.21203/rs.3.rs-86238/v1

License: (c) (1) This work is licensed under a Creative Commons Attribution 4.0 International License. Read Full License 
1 Driving resumption after critical illness: A survey and thematic analysis of patient experience and

2

$7 \quad{ }^{1}$ Critical Care Research Group, Guy's and St. Thomas' NHS Foundation Trust, London, United

8 Kingdom

$9 \quad{ }^{2}$ University of Hertfordshire, Hatfield, Hertfordshire, United Kingdom

$10{ }^{3}$ East \& North Herts NHS Trust, Stevenage, United Kingdom

$11{ }^{4}$ Florence Nightingale Foundation, London, United Kingdom

$12{ }^{5}$ GKT School of Medical Education, Faculty of Life Sciences and Medicine, King's College London,

13 London, United Kingdom

14

${ }^{6}$ Royal Berkshire NHS Foundation Trust, Reading, United Kingdom

15

\section{Corresponding Author:}

17

Joel Meyer, Critical Care, St. Thomas' Hospital, Westminster Bridge Rd, London SE1 7EH, United

18 Kingdom.

19

Email: Joel.Meyer@gstt.nhs.uk 
23 Joel Meyer: https://orcid.org/0000-0003-3303-3514

24 Natalie Pattison: $\underline{\text { https://orcid.org/0000-0002-6771-8733 }}$

25 Chloe Apps: https://orcid.org/0000-0002-4855-4243

26 Melanie Gager: https://orchid.org/0000-0001-5394-9314

27 Carl Waldmann: https://orcid.org/0000-0001-6643-5072 
Background: Critical care sequelae are common in survivors of critical illness. Physical, psychological and cognitive impairments can affect quality of life for years after the original insult. Driving is an advanced task reliant on complex physical and cognitive functioning. Driving represents a positive recovery milestone. Little is currently known about the driving habits of critical care survivors. The aim of this study was to explore the driving practices of individuals after critical illness. Methods: A purpose-designed questionnaire was distributed to critical care recovery clinic attendees at the 1year post hospital discharge time point. Results: A response rate of $90 \%$ was achieved. All respondents declared their intention to return driving. $68 \%$ had resumed driving by 3 months, $77 \%$ by 6 months and less than $80 \%$ by 1 year. The median interval (range) between critical care discharge and resumption of driving was 8 weeks ( 1 to 52 weeks). Psychological, physical and cognitive barriers were cited by respondents as barriers to driving resumption. Eight themes around driving resumption were identified through thematic framework content analysis; These were Confidence; Emotional strength/nervousness and anxiety; Concentration; Weakness and fatigue; Physical ability; Intrinsic motivation; Information; Timescales. Conclusion: This study demonstrates that resumption of driving following critical illness is substantially delayed. Qualitative analysis identified potentially modifiable barriers to driving resumption.

Keywords: Driving, Critical illness, Critical Care, Recovery, Intensive care

\section{Background}

Physical, psychological and cognitive impairments are common in survivors of critical illness [1].

These impairments, collectively known as Post-Intensive Care Syndrome (PICS), diminish quality of life and are burdensome for patients, carers and society [2, 3]. An increasing awareness and 
recognition of PICS has prompted commitments to enhance multidisciplinary aftercare with the overall goal of improving patient-centred outcomes and health-related quality of life.

Resumption of driving represents a crucial recovery milestone. For those patients who are licence holders, return to driving may promote independence and enable other determinants of recovery such as social activity and return to employment. Driving is, however, a complex task necessitating intact cognition (executive skills, visual perception, attention, memory and comprehension), physical ability (strength, sensation, coordination and reaction speed) as well as emotional preparedness. These elements may all be affected after critical illness.

Currently, continued driving eligibility is determined by medical diagnosis rather than capability to drive. National and international guidance regarding fitness to drive does not address the post intensive care period. Thus, healthcare professionals and individuals lack consistent advice as to when driving resumption can occur. Individuals may self-determine suitability to drive without professional guidance. Premature resumption carries risks to both self and other road users. Conversely, return to driving should not be arbitrarily prohibited or unduly prolonged. Enabling return to driving is important as it allows independence and return to everyday activities. Very little evidence exists to guide clinicians and patients following critical illness. Wolfe and Lehockey (2016) suggest clinicians should take into account functional ability and medical history, including conditions that may impair ability to drive and react, and correlate these factors with the risk for driving accidents [4].

Presently, little is known about when, or if, patients return to driving after critical illness and what barriers patients experience in relation to this activity. The aim of this study was to explore and gain insight into driving practices of individuals following critical illness. 
Design

77

An anonymised, cross-sectional, questionnaire was used to ascertain both quantitative (age, gender, driving status, time course of driving resumption) and qualitative experiential aspects of driving resumption (via open-ended questions). The primary outcome was the prevalence of driving resumption at 3 months following ICU discharge.

\section{Setting}

This study was undertaken in the adult critical care department of a large UK District General Hospital with 18 beds, approximately 800 admissions per year, ICU mortality of $18.9 \%$, and mixed medical/surgical case mix.

\section{Respondents}

Driving licence-holding adults having undergone 4 or more days mechanical ventilation attending the Rehabilitation After Critical IIIness outpatient clinic approximately 3, 6 and 12 months after hospital discharge were eligible to participate.

Fifty consecutive clinic attendees between 2014 and 2015 were invited to complete a short, anonymised questionnaire to solicit quantitative (timing) and qualitative data (open-ended textual responses) about driving resumption. The study was confirmed as a service evaluation that did not require ethical approval (UK Health Research Authority, http://www.hra.nhs.uk/researchcommunity/before-you-apply/determine-whether-your-study-is-research/). Informed consent was sought from all respondents. 
Survey questions were devised by an iterative process and were considered for content and face validity by two researchers (JM and $\mathrm{CW}$ ). Five questions were included in the final questionnaire (listed in Additional File 1). Respondents could opt to complete the handwritten paper survey whilst in clinic or alternatively in their own time by postal return. Respondents received assurance that any ongoing treatment or care would not be affected by participating in the survey.

\section{Data Analysis}

Quantitative data were of the nominal/ordinal form and analysed using SPSS Version 25 using descriptive frequency analyses (percentages). Continuous data were reported as a median and range. The Kaplan Meier method was used to analyse time-to-event i.e. days from ICU discharge to driving resumption.

Qualitative textual data from the open-ended questions were analysed by thematic framework analysis [5]. Themes for the framework were derived from the data, with the framework refined as data analysis took place. Data were examined by two researchers to ensure consensus was reached across each theme and how comments were attributed under those themes. These two researchers transcribed and analysed the handwritten survey data independently before agreeing the key themes for the framework analysis. This was then applied across all the data. A third researcher independently reviewed the raw data and choice of themes in the framework to increase robustness and reduce potential bias.

\section{Results}

45 out of 50 respondents completed the questionnaire ( $90 \%$ response rate). The median age of respondents was 60 years (range $27-82$ ), and 50\% female., All respondents possessed a valid driving 
122 licence and declared an intention to resume driving. The surveyed population was reflective of the

123 diverse critical care unit admission source: emergency admission 39\%, post-operative admission

$12425 \%$, medical inpatient ward admission $29 \%$, other $7 \%$.

125 The primary outcome, the proportion of respondents who had resumed driving by the 3-month

126 time-point after ICU discharge, was 68\%. By the 6-month time-point the proportion was $77 \%$.

127 The median interval between ICU discharge and resumption of driving was 8 weeks. The time period

128 for resumption was markedly variable ranging from 1 to 52 weeks post ICU discharge (Figure 1).

129 Over $20 \%$ had not resumed driving by the 1-year time-point.

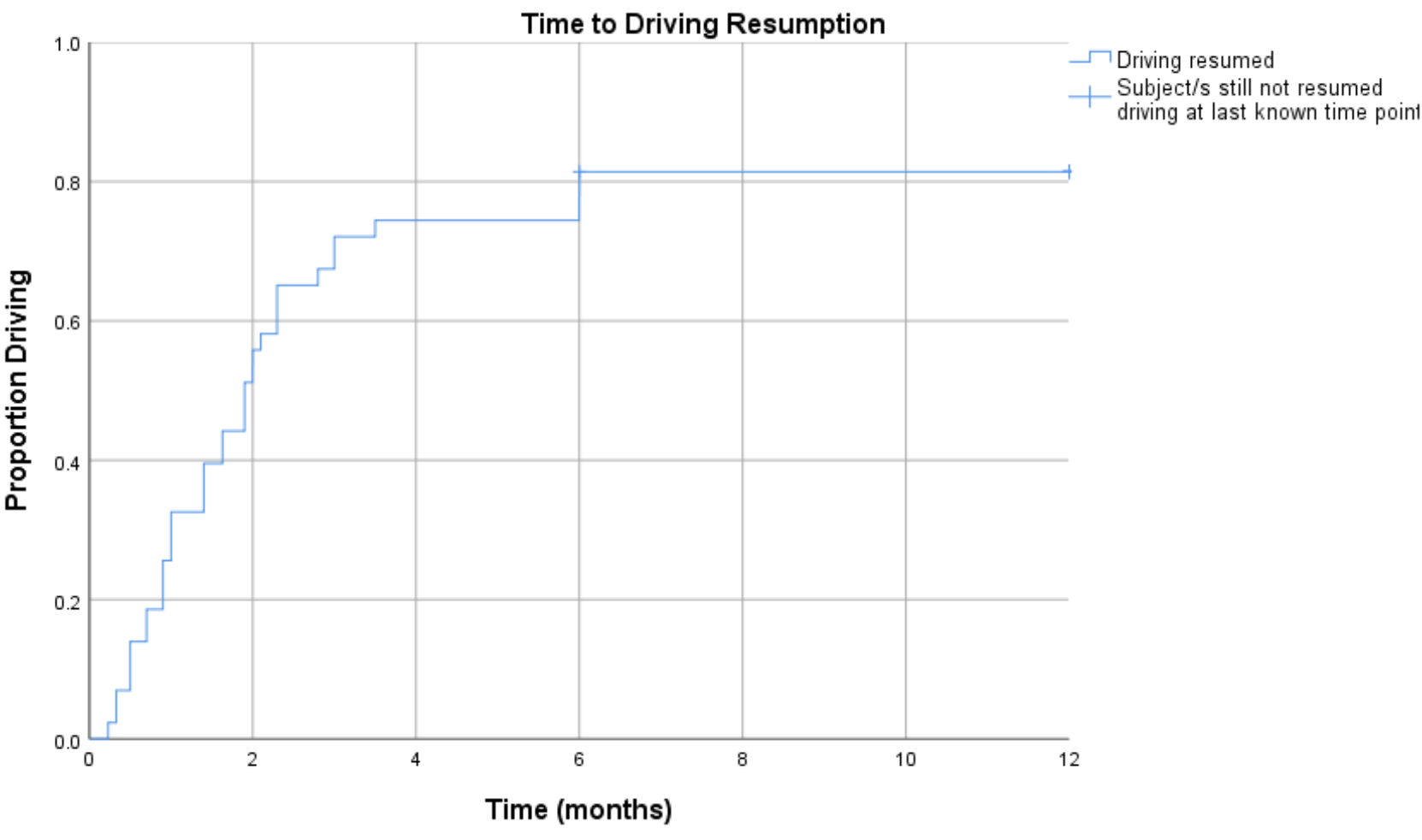


36 out of the 45 respondents provided information about the advice they received regarding driving resumption (Table 1). $22 \%$ did not receive any advice. Of the $78 \%$ that did, the GP was the most commonly cited source for information (22\%). DVLA and specialist consultants were cited by $11 \%$ of respondents. Other sources were nursing staff (6\%), the Rehabilitation After Critical Illness clinic (3\%) and insurance companies (3\%). One respondent had undertaken a 'return to driving' course. $19 \%$ of respondents reported that ability to perform an emergency stop was a key factor determining whether, or not, to resume driving.

\begin{tabular}{|l|c|}
\hline Sources of advice & No: of respondents \\
\hline Did not seek advice & 8 \\
\hline GP & 8 \\
\hline DVLA & 4 \\
\hline Specialist & 4 \\
\hline Nurse & 2 \\
\hline Recovery After Critical Illness clinic & 1 \\
\hline Insurance & 1 \\
\hline Return to driving course & 1 \\
\hline Emergency stop & 7 \\
\hline
\end{tabular}

Qualitative analysis

147 Eight principal themes regarding driving resumption were identified from the framework analysis

148 (Table 2). These were; Confidence; Emotional strength/nervousness and anxiety; Concentration;

149 Weakness and fatigue; Physical ability; Intrinsic motivation; Information; Timescales. 
151 Confidence

152 Lack of confidence determined how and when people returned to driving. Various strategies were

153 used to mitigate against this, including having accompanied practice. Some respondents suggested

154 'driving buddies' and with many advocating local drives first. Confidence was reported to increase

155 once driving had been attempted and, in most cases, helped contribute to an overall sense of

156 recovery.

157

"... started with short trips of less than 1 mile, which gradually got longer. Accompanied

158

159 practice, a 'driving buddy' was useful for confidence" (Respondent 3)

"I did a test drive to check spatial awareness etc.." (Respondent 8)

160

161

Emotional strength/nervousness and anxiety

162

Nervousness was cited as a factor related to confidence;

163

"I found it stressful at first" (Respondent 10)

164

"I didn't want to rush and put my family in danger" (Respondent 5)

165

One respondent mentioned developing anger when driving highlighting emotional lability as a

166 feature in driving ability.

167

168

Concentration

169

Feeling muddled, mentally unprepared and reduced concentration were reported and related to

170 slower response times and decreased mental alertness. This theme also related to spatial 
awareness, and one driver reported having a minor accident on their first drive since discharge. Respondents varied in their self-awareness of their mental capacity to drive.

Weakness and fatigue were cited by respondents and these underpinned other themes. concentration" (Respondent 13) too tired to drive more than 1 hour" (Respondent 2) (Respondent 3)

The physical ability to drive and perform certain driving functions, such as emergency stops, was a determining factor in driving resumption. For some respondents this aspect prevented driving for several months.

"I waited until legs felt strong....able to use brake in emergency" (Respondent 6) be useful. 
Intrinsic motivation seemed to be a significant factor for many respondents. Self-motivation is important in resuming normal activities and return to driving was used as a marker and milestone of recovery by respondents. Driving appeared to be a defining activity in people's recovery and their sense of self-worth. "... (driving is) important for 'returning to normal'" (Respondent 16) "Desire to get my independence back" (Respondent 23)

Information

202 The source of information accessed by individuals about driving following critical illness was highly variable. Respondents reported that advice from GPs often focused on physical ability, and in particular, ability to undertake an emergency stop was commonly used as a proxy for readiness to drive. However mental and cognitive ability were not interrogated. "No guidance - doctor said 'when I felt ready'" (Respondent 16)

211 Timescales for those who returned to driving varied significantly, with feelings of being 'ready' most commonly cited by respondents, thus suggesting a need for mental preparedness. 
216 Respondent-generated ideas to assist with driving resumption included driving re-tests, time with

217 driving instructors to build confidence and motorway driving courses. Graded exposure to driving was a recurring theme, with many people initially 'testing' their ability to drive safely by undertaking short distance journeys. Clear accessible guidance after critical illness was advocated by respondents.

\section{Discussion}

This single centre study elicited the incidence, time-course and experience of driving resumption among 45 adult critical illness survivors. The findings highlight that driving resumption is often delayed in critical care survivors with less than $80 \%$ returning to drive one-year post discharge in our study findings. Many individuals rely on driving as a means of accessing work and social support, especially in rural areas and communities. Approximately one million people in the UK and over three million people in the US are primarily employed as drivers or are required to drive as part of their job, reflecting the importance of driving after critical illness to the economy as well as individual financial stability [6, 7].

Driving enables resumption of other important activities alongside work. Critical illness survivors often have on-going health requirements and for ease of access a vehicle is frequently required. Accessing hobbies and leisure pursuits are vital for enhancing quality of life [8]. Driving enables parental and carer responsibilities often required of individuals in this demographic. As noted in our study, driving was a defining activity in people's recovery and their sense of self-worth. This echoes findings from previous research highlighting the desire to regain independence following critical illness [9]. 
An unnecessary delay in driving resumption could impact the mental health of these individuals, further impeding recovery. Isolation and depression have been noted in older adults who have experienced a similar loss of independence [10-13]. Commentary from individuals in the public domain describe how in old age the car begins to represent life with feelings of freedom and normality being restored, even if only temporarily. The nature of not being able to drive represents an unwelcome agonising change [14]. Frailty associated with critical care survivorship may mean that individuals of a lower age demographic may entertain similar thoughts and feelings. Numerous barriers to driving resumption were cited by respondents. Lack of confidence was a highlighted factor. Respondents in this study often cited accompanied and local drives to rebuild confidence and enable return to driving. Only one respondent underwent a return to driving course, but this may be an avenue for survivors to explore to enable safe and timely resumption of this activity.

There is a clinical responsibility too that needs to be considered. From whom, and how, these survivors gain their information about driving resumption is likely to be highly variable. Clinicians need to consider the cognitive, and especially the executive functioning level, alongside the functional ability, which is often the primary focus. Medications used to support critical illness recovery, may indeed impair ability to drive. A comprehensive approach to assessment is required, and in cases that are difficult to determine ability in the cognitive domain, a further neuropsychiatric/psychological assessment may be needed [4]. Ability to perform an emergency stop is not included in any guidance or standards, however from our survey it appears to be used as a proxy by healthcare professionals and patients in determining readiness to resume driving. specific to driving after critical illness. Limitations of our study include the single-centre methodology which may not reflect the UK-wide or global picture. Clinical data about type/severity of critical 

illness were not collected. Strengths of our study include the high response rate and the wealth of qualitative data ascertained relative to survey length.

264

\section{Conclusion}

266 Inconsistency of information received by respondents about driving resumption after critical illness

267 in this study reflects lack of published guidance. Although the deleterious effects of a critical care

268 stay are well documented [15] research about the impact these have on subsequent driving ability is

269 lacking. Reducing avoidable delay to driving should be viewed as a low-cost high impact intervention

270 to enhance health related quality of life. Further research is needed to understand the

271 epidemiology, enablers and barriers to driving in this population.

List of abbreviations

274

PICS - Post-intensive care syndrome

275 
Table 2: Evidence of themes

\begin{tabular}{|c|c|c|}
\hline Themes & $\begin{array}{l}\text { Number of } \\
\text { comments } \\
\text { made }\end{array}$ & Sample comments \\
\hline Timescales & 40 & $\begin{array}{l}\text { Respondent } 1 \text { - Female, 68: “Approx. } 3 \text { months (1st time)" } \\
\text { Respondent } 29 \text { - Male, 60: "At least } 10 \text { weeks" }\end{array}$ \\
\hline Information & 34 & $\begin{array}{l}\text { Respondent } 8 \text { - Male, 75: "Asked ward doctor (concerned re: medication side effects); advised if could } \\
\text { brake, okay" } \\
\text { Respondent } 23 \text { - Female, 36: "Discussed with work and GP; GP advised against it at first. Course } \\
\text { arranged via work. No information given from hospital (how/when/legalities)." }\end{array}$ \\
\hline Physical Ability & 20 & $\begin{array}{l}\text { Respondent } 5 \text { - Male, 34: "Not strong enough at first; waited until gained some weight" } \\
\text { Respondent } 9 \text { - Female, 31: "Hard work in arms; waited until I could do an emergency stop; had an } \\
\text { open wound (covered) - waited until it didn't hurt" }\end{array}$ \\
\hline Confidence & 26 & $\begin{array}{l}\text { Respondent } 21 \text { - Male, 76: "Confidence an important factor (lack of) after long hospital stay" } \\
\text { Respondent } 42 \text { - Male, 64: "Apprehensive at first; start/ed when confident in self" }\end{array}$ \\
\hline Intrinsic motivation & 24 & Respondent 13 - Female, 38: “Need to be mentally 'ready' \\
\hline
\end{tabular}




\begin{tabular}{|c|c|l|}
\hline & & Respondent 16 - Male, 66: "Important for 'return to normal'" \\
\hline Emotional Strength/ Nervousness & 15 & Respondent 15 - Female, 72: "Felt so vulnerable (so waited longer than advised)" \\
and Anxiety & Respondent 19 - Female, 55: "Wanted to return to being the person I was before all this trauma" \\
\hline Concentration & 11 & $\begin{array}{l}\text { Respondent 12 - Female, 75: "Extremely muddled after discharge; more alert now" } \\
\text { Respondent 36 - Male, 61: "Mental ability problem; information processing; things move too fast after } \\
\text { a long period of not driving" }\end{array}$ \\
\hline Weakness/Fatigue & $\begin{array}{l}\text { Respondent 7 - Female, 44: "Needed to feel stronger (for long journeys)" } \\
\text { Respondent 37 - Male, 71: "Felt weak and listless with no energy and not until 5/6 weeks that felt } \\
\text { able" }\end{array}$ \\
\hline
\end{tabular}




\section{Declarations}

\section{Ethics approval}

The study was confirmed as a service evaluation that did not require ethical approval (UK Health Research Authority, http://www.hra.nhs.uk/research-community/before-you-apply/determinewhether-your-study-is-research/).

\section{Consent to participate}

Informed consent was obtained from all individual respondents included in the study.

\section{Consent for publication}

Not applicable

\section{Availability of data and material}

The survey data that support the findings of this study are available from the corresponding author, (JM), upon reasonable request.

\section{Competing interests}

The author(s) declare no potential conflict of interest with respect to the research, authorship, and/or publication of this article. 


\section{Funding}

The author(s) received no financial support for the research, authorship, and/or publication of this article.

\section{Authors contributions}

$\mathrm{JM}, \mathrm{CW}, \mathrm{NP}$ and MG were responsible for study conception and design. CA and JM were responsible for quantitative data analysis and interpretation. NP and JM were responsible for qualitative data analysis and interpretation. JM and CA prepared the manuscript. All authors contributed to manuscript revision and approved the final version for submission. JM acts as the guarantor for the intellectual integrity of the data.

\section{Acknowledgements}

Not applicable 


\section{References}

1. Connolly, B., et al., Physical rehabilitation interventions for adult patients during critical illness: an overview of systematic reviews. Thorax, 2016. 71(10): p. 881-90.

2. Cuthbertson, B.H., et al., Quality of life in the five years after intensive care: a cohort study. Critical care (London, England), 2010. 14(1): p. R6-R6.

3. Herridge, M.S., et al., Functional Disability 5 Years after Acute Respiratory Distress Syndrome. New England Journal of Medicine, 2011. 364(14): p. 1293-1304.

4. Wolfe, P.L. and K.A. Lehockey, Neuropsychological Assessment of Driving Capacity. Arch Clin Neuropsychol, 2016. 31(6): p. 517-29.

5. Ritchie, J. \& Spencer, L. 1994. Qualitative data analysis for applied policy research by Jane Ritchie and Liz Spencer in A. Bryman and R. G. Burgess [eds.] 'Analysing qualitative data', (pp.173-194). London: Routledge.

6. Department for Transport. GB Driving Licence Data. 201902 October 2019]; Available from: https://data.gov.uk/dataset/d0be1ed2-9907-4ec4-b552-c048f6aec16a/gb-driving-licencedata.

7. U.S Census Bureau. American Community Survey 2017. 2019 [cited 20208 January]; Available from: https://www.census.gov/data/tables/2017/demo/industryoccupation/truckers-acs17.html.

8. Hashem, M.D., et al., Patient outcomes after critical illness: a systematic review of qualitative studies following hospital discharge. Critical Care, 2016. 20(1): p. 345.

9. Ågård, A.S., et al., Struggling for independence: A grounded theory study on convalescence of ICU survivors 12 months post ICU discharge. Intensive and Critical Care Nursing, 2012. 28(2): p. $105-113$.

10. Marottoli, R.A., Mendes de Leon, C. F., Glass, T. A., Williams, C. S., Cooney, L. M., Jr, Berkman, L. F., \& Tinetti, M. E., Driving cessation and increased depressive symptoms: 
prospective evidence from the New Haven EPESE. Journal of the American Geriatrics Society, 1997. 45: p. 202-206.

11. Windsor, T.D., et al., The Role of Perceived Control in Explaining Depressive Symptoms Associated With Driving Cessation in a Longitudinal Study. The Gerontologist, 2007. 47(2): p. 215-223.

12. Marottoli, R.A., et al., Consequences of Driving Cessation: Decreased Out-of-Home Activity Levels. The Journals of Gerontology: Series B, 2000. 55(6): p. S334-S340.

13. Mezuk, B. and G.W. Rebok, Social Integration and Social Support Among Older Adults Following Driving Cessation. The Journals of Gerontology: Series B, 2008. 63(5): p. S298S303.

14. Aronson, L., Don't Ruin My Life - Aging and Driving in the 21st Century. N Engl J Med, 2019. 380(8): p. 705-707.

15. Azoulay, E., et al., Recovery after critical illness: putting the puzzle together-a consensus of 29. Crit Care, 2017. 21(1): p. 296. 
Additional File 1

Driving After Intensive Care Survey

Additional File 2

Framework Analysis for Driving after Critical Illness 
Figures

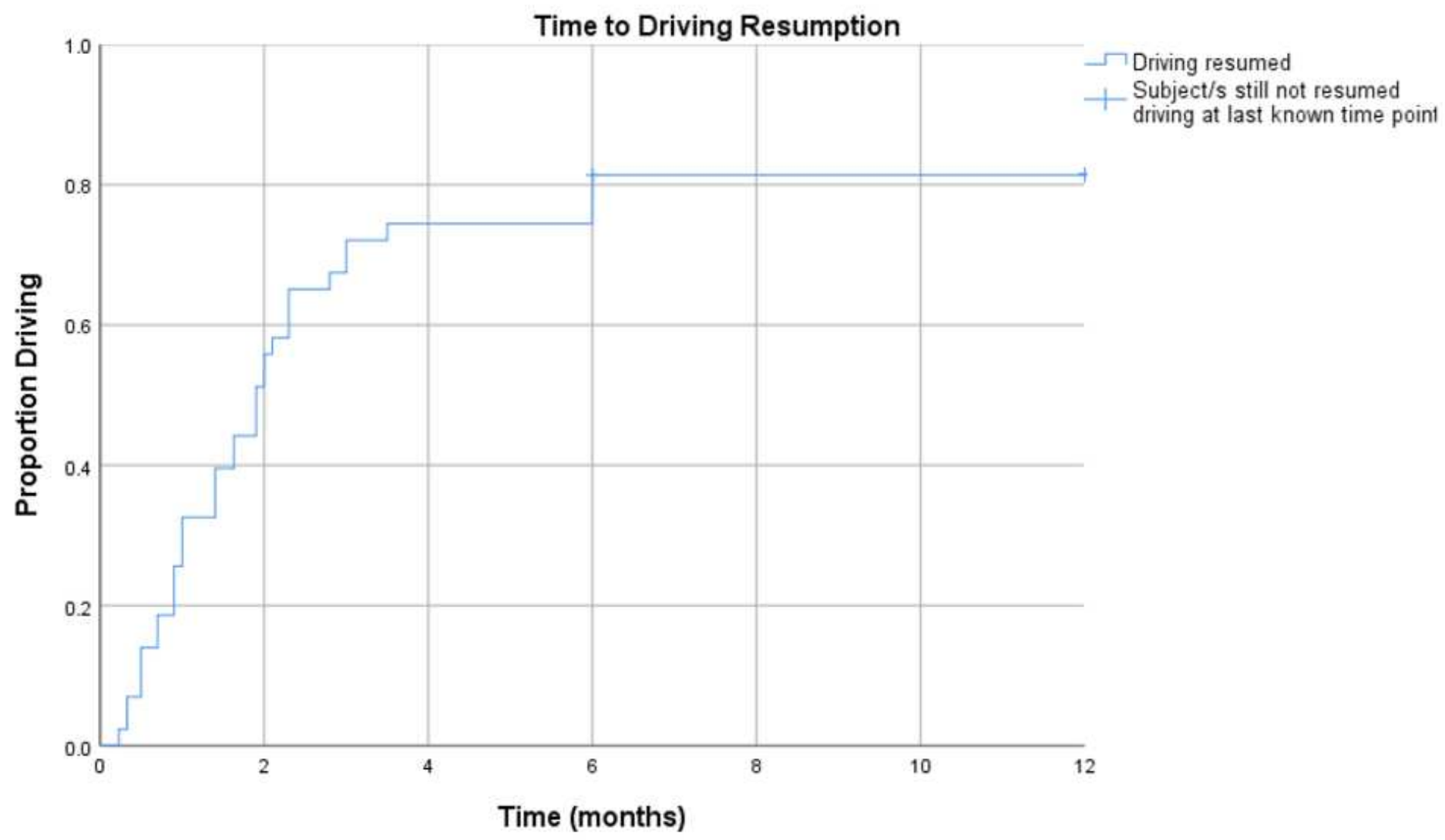

Figure 1

Kaplan-Meier curve time to driving resumption after ICU discharge

\section{Supplementary Files}

This is a list of supplementary files associated with this preprint. Click to download.

- AdditionalFile1CRITICALCARE.pdf

- AdditionalFile2FrameworkanalysisCRITICALCARE.pdf 